\title{
Hydration Water Dynamics in Bovine Serum Albumin at Low Temperatures as Studied by Deuterium Solid-State NMR
}

Tatsuya Miyatou, Takashi Araya, Ryutaro Ohashi, Tomonori Ida, Motohiro Mizuno*

${ }^{I}$ Department of Chemistry, Graduate School of Natural Science and Technology, Kanazawa University, Kakuma-machi Kanazawa, Ishikawa 920-1192, Japan

Corresponding author. tel.: +81 76264 5686; fax: +81 762645742 .

E-mail address: mizuno@se.kanazawa-u.ac.jp (M. Mizuno) 


\section{ABSTRACT}

Solid state ${ }^{2} \mathrm{H}$ NMR was used to investigate changes in the structure and dynamics of hydration waters of bovine serum albumin (BSA) due to glass transitions. The ${ }^{2} \mathrm{H}$ NMR spectra were separated into fast and slow components based on differences in spin-lattice relaxation time $\left(T_{1}\right)$. The fast components corresponded to water molecules interacting with protein while the slow components were the water molecules similar to bulk water and deuterons of the protein backbone. Simulation analysis of the ${ }^{2} \mathrm{H}$ NMR spectra of the fast components was used to assess the mode and rate of motions of hydration waters around the protein. At low temperatures, the water molecules underwent a $180^{\circ}$ flip and slow reorientation in the tetrahedral sites. The distribution of the rate of the $180^{\circ}$ flip and the D-O-D angle of water molecules were clarified. The distribution of the D-O-D angle of water molecules spread with decreasing temperature. The marked slowing down in the reorientation of water molecules was observed at a glass transition of around $200 \mathrm{~K}$, which is linked to the disordered region of the protein. In contrast, the $180^{\circ}$ flip of water molecules occurred frequently, even below $200 \mathrm{~K}$. A freeze of the $180^{\circ}$ flip of water molecules was observed around the glass transition temperature of $110 \mathrm{~K}$, where primary hydrate water formed a direct hydrogen bond with the protein, making it perfectly immobile. 


\section{INTRODUCTION}

The changes in the structure and dynamics of proteins are caused by glass transition and these changes are directly related to the biological activity of proteins [1-4].

The glass transition of bovine serum albumin (BSA) has been investigated by thermal analysis [5,6]. A sample quenched from $300 \mathrm{~K}$ down to $80 \mathrm{~K}$ showed a jump in heat capacity indicating a glass transition temperature $T_{\mathrm{g}}$ of $170 \mathrm{~K}$. When the sample was annealed at $200-240 \mathrm{~K}$, the relaxation effects resulted in three glass transition temperatures: $T_{\mathrm{g}}=110,135$ and $>180 \mathrm{~K}$. The glass transition above $180 \mathrm{~K}$ is considered to originate from a rearrangement in the motion of the disordered region of the protein. Based on dielectric measurements, the corresponding glass transition was observed at $200 \mathrm{~K}[7,8]$. The transition accompanying the change in the dynamics of protein and hydration water, also known as the dynamic transition, was found at about $200 \mathrm{~K}$ [1-4]. The glass transitions at $T_{\mathrm{g}}=110$ and $135 \mathrm{~K}$ are considered to be caused by a rearrangement in the motions of primary hydrate water forming a direct hydrogen bond with the protein and part of the internal water localized in the opening of a protein's structure, respectively. Thus, for hydrated proteins, the dynamics of hydration waters plays very important roles in the glass transition. Therefore, detailed analysis of the dynamics of hydration waters around the glass transition is important to investigate the physical properties of proteins. Previously, the relations between the dynamics of hydration waters and the glass or dynamical transition were 
investigated by various methods [7-16].

NMR is an effective method to study molecular dynamics and local structures in hydrated proteins [17-29]. There have been several investigations of the structure and dynamics of BSA using NMR [26,29]. To investigate the dynamics of water molecules, solid-state ${ }^{2} \mathrm{H}$ NMR is especially efficient, since the rate and mode of reorientational motions of molecules can be analyzed strictly from the line shape of a broadline spectrum and $T_{1}$ [30-40]. Thus far, the dynamic properties of hydration waters in elastin, collagen, and myoglobin have been analyzed using ${ }^{2} \mathrm{H}$ NMR $\left(T_{1}\right)$ [41-43]. Although the deuteration causes a slight change in its bonding properties, the glass transition behavior and dynamics of water were little affected by deuteration [16]. In the present work, we tried to apply simulation analysis of solid-state ${ }^{2} \mathrm{H}$ NMR spectra to BSA to obtain more detailed information of the dynamics and structure of hydration waters and to clarify the relations between these two properties and glass transitions. Simulation of ${ }^{2} \mathrm{H}$ NMR broadline spectra, including distribution of the motions of water molecules, was performed. Changes in the motional mode, rate and D-O-D angles of hydration waters in BSA with decreasing temperature were investigated using the simulation analysis of ${ }^{2} \mathrm{H}$ NMR broadline spectra and $T_{1}$.

\section{MATERIALS AND METHODS}


BSA was obtained from Wako Pure Chemical Industries. BSA powder hydrated with deuterium oxide was prepared by repeated recrystallization three times from heavy water. The hydration level of samples was determined from the observed mass change of dried samples. Although the contents of small particles such as salt ions potentially present may give uncertainness, the hydration level of samples was estimated as $h=0.26$ (h: gram of water per gram of protein).

The ${ }^{2} \mathrm{H}$ NMR spectra and $T_{1}$ were measured by using a Chemagnetics CMX-300 spectrometer at $45.825 \mathrm{MHz}$. The sample temperature was controlled with a nitrogen-gas-flow temperature controller (JEOL VT1A). The sample was sealed in a glass tube $6 \mathrm{~mm}$ in diameter and about 20 $\mathrm{mm}$ in length. The ${ }^{2} \mathrm{H}$ NMR spectra were observed using a quadrupole echo sequence $\left(90^{\circ}\right)_{x}-t$ $-\left(90^{\circ}\right)_{y}-t-t_{\text {acq }}$, where $t$ and $t_{\text {acq }}$ are the interval of echo and acquisition time, respectively. The $90^{\circ}$ pulse width and $t$ were 2.7 and $20 \mu \mathrm{s}$, respectively. The partially relaxed spectra for saturation recovery were observed using a sequence $\left(90^{\circ}-t_{\mathrm{s}}\right)_{\mathrm{n}}-90^{\circ}-t_{r}-\left(90^{\circ}\right)_{x}-t-\left(90^{\circ}\right)_{y}-t-$ $t_{\text {acq. }}$ n was $5 . t_{\mathrm{s}}, t$ and $t_{\text {acq }}$ were 100,20 and $2048 \mu$ s, respectively. ${ }^{2} \mathrm{H}$ NMR $T_{1}$ was measured by the inversion recovery method. The simulation of ${ }^{2} \mathrm{H}$ NMR partially relaxed spectra was performed by homemade Fortran programs using double precision [39].

\section{RESULTS AND DISCUSSION}




\section{${ }^{2} \mathrm{H}$ NMR spectra and $T_{1}$}

Fig. 1 shows the temperature dependence of the ${ }^{2} \mathrm{H}$ NMR broadline spectrum. Above $273 \mathrm{~K}$, a sharp spectrum due to the rapid isotropic rotation of water molecules was mainly observed. Below $273 \mathrm{~K}$, a broad component with peaks at about $\pm 60 \mathrm{kHz}$ due to the freezing of the motions of hydration waters appeared in the spectrum and its intensity increased with decreasing temperature. The line width of the central component increased with decreasing temperature. The broad central component below $213 \mathrm{~K}$ indicates the existence of the $180^{\circ}$ flip of water molecules representing the distribution of the D-O-D angle. The central component of the spectrum disappeared and the ${ }^{2} \mathrm{H}$ NMR spectrum became an almost rigid powder pattern below $T_{\mathrm{g}}=110 \mathrm{~K}$ accompanied by the freezing of primary hydrate water.

Fig. 2 shows partially relaxed ${ }^{2} \mathrm{H}$ NMR spectra for the inversion recovery method at $203 \mathrm{~K}$. As shown in the spectrum at a recovery time of $t_{r}=10 \mathrm{~ms}$, recovery of the central sharp component was faster than that of the broad component. Fig. 3 shows the recovery of magnetization for the inversion recovery method. The recovery of magnetization was analyzed by two-component fitting using

$$
M\left(t_{r}\right) / M_{0}=A_{f}\left[1-2 \alpha \exp \left(\left(-t_{r} / T_{1 f}\right)^{\beta_{f}}\right)\right]+\left(1-A_{f}\right)\left[1-2 \alpha \exp \left(-\left(t_{r} / T_{1 s}\right)^{\beta_{s}}\right)\right] .
$$

Here, $M\left(t_{r}\right)$ is the magnetization at recovery time $t_{r}$ and $M_{0}$ is the thermal-equilibrium value of magnetization. $A_{f}$ is the ratio of the fast component. $T_{1 f}, \beta_{f}$ and $T_{1 s}, \beta_{s}$ are the spin-lattice 
relaxation times and the stretching parameters for fast and slow components, respectively. $\alpha$ shows imperfection in the $180^{\circ}$ pulse. The deuterated parts in the protein backbone as well as water molecules undergoing slow motion are considered to be included in the slow component.

Only one component was observed below $160 \mathrm{~K}$. The mean $T_{1 f}$ and $T_{1 s}$ values were obtained by [41-43]

$$
\left\langle T_{1 i}\right\rangle=\frac{T_{1 i}}{\beta_{i}} \Gamma\left(\frac{1}{\beta_{i}}\right), \quad(i=f, s)
$$

where $\Gamma(x)$ is the Gamma function.

Fig. 4 shows the temperature dependence of $\left\langle T_{1}\right\rangle$ for fast and slow components. $\left\langle T_{1 s}\right\rangle$ increased with decreasing temperature. The minimum of $\left\langle T_{1 f}\right\rangle$ was observed around $230 \mathrm{~K}$. $\left\langle T_{1 f}\right\rangle$ is predicted to be dominated by the isotropic rotation of water molecules. For the isotropic rotation of water molecules, ${ }^{2} \mathrm{H}$ NMR $T_{1}$ is written as

$$
T_{1}^{-1}=\frac{3 \pi^{2}}{10}\left(1+\frac{\eta^{2}}{3}\right)\left(\frac{e^{2} q Q}{h}\right)^{2}\{J(\omega)+4 J(2 \omega)\}
$$

where $e^{2} q Q / h$ and $\eta$ are quadrupole coupling constant and asymmetry parameter, respectively. $J(\omega)$ is spectral density and is the Fourier transform of an autocorrelation function $G(\tau)$ which reflects the motions of water molecules,

$$
J(\omega)=\int G(\tau) \exp (-i \omega t) d t
$$

When $G(\tau)$ is represented as an exponential function, $G(\tau)=\exp (-t / \tau), J(\omega)$ can be written as the BPP form [44]: 


$$
J(\omega)=\frac{\tau}{1+(\omega \tau)^{2}}
$$

Here, $\omega$ and $\tau$ are ${ }^{2} \mathrm{H}$ NMR frequency and correlation time, respectively. The minimum value of $T_{1}$ was estimated to be $1.5 \times 10^{-3} \mathrm{~s}$ from eqs. (3) and (5) using the quadrupole interaction parameters of $e^{2} q Q / h=230 \mathrm{kHz}$ and $\eta=0$ which were obtained from the line shape of the ${ }^{2} \mathrm{H}$ NMR spectrum at low temperatures. The observed $\left\langle T_{1 f}\right\rangle$ minimum $\left(5 \times 10^{-3} \mathrm{~s}\right)$ was longer than this calculated value. This result suggests the distribution of the correlation time $\tau$. When a distribution of correlation time exists, $J(\omega)$ is well described by the Cole-Cole spectral density [45]:

$$
J(\omega)=\frac{\sin \left(\frac{\pi}{2} \alpha_{\mathrm{cc}}\right)\left(\omega \tau_{\mathrm{cc}}\right)^{\alpha_{\mathrm{cc}}}}{\omega\left(1+\left(\omega \tau_{\mathrm{cc}}\right)^{2 \alpha_{\mathrm{cc}}}+2 \cos \left(\frac{\pi}{2} \alpha_{\mathrm{cc}}\right)\left(\omega \tau_{\mathrm{cc}}\right)^{\alpha_{\mathrm{cc}}}\right)}
$$

where $\tau_{\mathrm{cc}}$ and $\alpha_{\mathrm{cc}}$ are the correlation time at the maximum position and the width of distribution, respectively. The distribution of correlation time $g_{\mathrm{cc}}(\tau)$ is written as

$$
g_{C C}(\tau)=\frac{\sin \left(\alpha_{C C} \pi\right)}{2 \pi\left[\cosh \left\{\alpha_{C C} \ln \left(\frac{\tau}{\tau_{C C}}\right)\right\}+\cos \left(\alpha_{C C} \pi\right)\right]} .
$$

From the minimum of $\left\langle T_{1 f}\right\rangle, \alpha_{\mathrm{cc}}=0.52$ was estimated. Using this $\alpha_{\mathrm{cc}}$ value and eqs. (3) and (6), $\tau_{\mathrm{cc}}$ was estimated from $\left\langle T_{1 f}\right\rangle$.

Fig. 5 shows the temperature dependence of $\tau_{\mathrm{cc}}$. An Arrhenius-type temperature dependence was observed for $\tau_{\mathrm{cc}}$. The change in the slope of $\log \left(\tau_{\mathrm{cc}}\right)$ vs. the $1 / T$ plot was observed around $200 \mathrm{~K}$. The activation energy of the motion of water molecules was obtained as 57 and 35 
$\mathrm{kJmol}^{-1}$ above and below $200 \mathrm{~K}$, respectively. The glass transition around $200 \mathrm{~K}$ was caused by the rearrangement motion of the disordered region in the protein $[1,2]$. Therefore, the dynamics of hydration waters is affected by freezing of the disordered region in the protein accompanied with a glass transition. The decrease in activation energy at low temperatures indicates a change of the motional mode of hydration water at around $200 \mathrm{~K}$.

\section{${ }^{2}$ H NMR partially relaxed spectrum}

In order to obtain the ${ }^{2} \mathrm{H}$ NMR spectrum of the fast component, the partially relaxed spectra for saturation recovery were measured. The spectrum of the fast component was selectively obtained by setting recovery time $\tau_{\mathrm{r}}$ after saturation pulses as $1 / 10$ of the $\left\langle T_{1}\right\rangle$ value of the slow

component. Fig. 6 shows the ${ }^{2} \mathrm{H}$ NMR spectrum of the fast component. The ${ }^{2} \mathrm{H}$ NMR spectrum of the fast component observed at $233 \mathrm{~K}$ showed a sharp peak due to isotropic rotation or a jump among the tetrahedral sites of the water molecule. The intensity of the broad component with a line width of $c a . \pm 60 \mathrm{kHz}$ increased with decreasing temperature. Moreover, the line width of the central portion of the spectrum broadened with decreasing temperature. Analysis of the ${ }^{2} \mathrm{H}$ NMR spectrum of the fast component was performed using spectral simulation.

The motions and angles of water molecules used for the simulation analysis are shown in Fig.

7. The combination of a $180^{\circ}$ flip and slow jump to the other two sites in the tetrahedral sites 
(i.e., reorientation of water molecules) was considered in the motions of water molecules. $k_{180^{\circ}}$ and $k_{\mathrm{re}}$ are the rates of the $180^{\circ}$ flip and the slow jump to the other two sites in the tetrahedral sites of water molecules, respectively in Fig. 7. $\theta$ is the angle between the electric field gradients in two deuterons of a water molecule and almost corresponds to the D-O-D angle except for intermolecular interactions. The distributions of $k_{180^{\circ}}, k_{\mathrm{re}}$ and $\theta$ were considered for the simulation of the ${ }^{2} \mathrm{H}$ NMR spectrum. The intensity of the partially relaxed spectrum depends on $T_{1}$ which is dominated by jumping rate $k_{180^{\circ}}$. The reduction factor $g_{\text {red }}\left(k_{180^{\circ}}\right)$ for the signal intensity due to partial relaxation is written as

$$
g_{\text {red }}\left(k_{180^{\circ}}\right)=1-\exp \left(-\frac{t_{r}}{T_{1}}\right)
$$

$T_{1}$ for the $180^{\circ}$ flip of water molecules was obtained by

$$
T_{1}^{-1}=\frac{9 \pi^{2}}{40}\left(\frac{e^{2} q Q}{h}\right)^{2}(\sin \theta)^{2}\left(\frac{\tau}{1+(\omega \tau)^{2}}+\frac{4 \tau}{1+(2 \omega \tau)^{2}}\right)
$$

where $\tau$ and $k_{180^{\circ}}$ are related as $k_{180^{\circ}}=(2 \tau)^{-1}$.

Each spectral component was multiplied by $g_{\text {red }}\left(k_{180^{\circ}}\right)$ in the simulation of the partially relaxed ${ }^{2} \mathrm{H}$ NMR spectrum. The distribution of $k_{180^{\circ}}$ was estimated using the distribution of correlation time $\tau$ obtained from $\left\langle T_{1 f}\right\rangle$. The Cole-Cole equation of eq. (7) with same distribution width as $k_{180^{\circ}}$ was used for the distribution of $k_{\mathrm{re}}$. The Gaussian function was assumed for the distribution of $\theta$. The distribution centers of $k_{\mathrm{re}}$ and $\theta$ and the distribution width of $\theta$ were determined from spectral fitting (Fig. 6). Fig. 8 shows the distribution of $k_{\mathrm{re}}, k_{180^{\circ}}$ and $\theta$. The center of $k_{\mathrm{re}}$ was 
lower than that of $k_{180^{\circ}}$ by about double digits. The effect of reorientation of water molecules on the spectrum was not observed in the spectrum at $173 \mathrm{~K}$. On the contrary, a large amount of water molecules underwent a $180^{\circ}$ flip with more than $10^{4} \mathrm{~Hz}$ even at $173 \mathrm{~K}$. Thus, the behavior of water molecules observed in the ${ }^{2} \mathrm{H}$ NMR spectra of the fast component was largely different from that of bulk waters [40]. The high mobility of unfreezeable water, which constructs a shell layer, and bound water, which attaches directly on the protein surface even at low temperatures, has been shown for BSA from dielectric measurements [13]. Therefore, the water molecules observed in the ${ }^{2} \mathrm{H}$ NMR spectra of the fast component are considered to be near to the protein and interacting with it. Although the motions of water molecules near protein are restricted and the reorientation of water molecules is suppressed by freezing of the disordered region in the protein due to a glass transition around $200 \mathrm{~K}$, those water molecules undergo a $180^{\circ}$ flip even below $200 \mathrm{~K}$. The rate of the $180^{\circ}$ flip of the water molecules near protein decreases with decreasing temperature. The rigid pattern of the ${ }^{2} \mathrm{H}$ NMR spectrum below $110 \mathrm{~K}$ (Fig. 1) indicates freezing of the $180^{\circ}$ flip of those water molecules. The decrease in the activation energy obtained from the temperature dependence of $\tau_{\mathrm{cc}}$ below $200 \mathrm{~K}$ corresponds to the change from the tetrahedral jump to the $180^{\circ}$ flip of the water molecules.

The distribution width of the D-O-D angle $(\theta)$ increased with decreasing temperature, as shown in Fig. 8. In fact, $\theta$ is the angle between the electric field gradients in two deuterons of a 
water molecule. Therefore, intermolecular interactions related to the hydrogen bond affect $\theta$.

The increase in the distribution of $\theta$ shows an increase in the interactions between water molecules and protein accompanied by a slowing down of motions of the water molecules.

\section{CONCLUSIONS}

In the present work, we clarified the motions and structures of the hydrate waters around low-temperature glass transitions. The isotropic rotation of water molecules near protein are restricted by freezing of the disordered region in the protein around the glass transition temperature $\left(T_{\mathrm{g}}=200 \mathrm{~K}\right)$ and the $180^{\circ}$ flip becomes a dominant motion for water molecules below $T_{\mathrm{g}}$. The gradual slowing down of the $180^{\circ}$ flip of water molecules near protein was observed in the temperature range of 200-110 K. The distribution width of the D-O-D angle of water molecules increased gradually by decreasing the rates of motions of water molecules at low temperatures. Freezing of the $180^{\circ}$ flip of water molecules near the protein was observed around another glass transition temperature $\left(T_{\mathrm{g}}=110 \mathrm{~K}\right)$.

\section{ACKNOWLEDGEMENT}

This work was supported by CREST, JST, JSPS KAKENHI Grant Numbers 26286002, 
19510103, 24651113 and Kanazawa University CHOZEN Project.

$-13-$ 


\section{REFERENCES}

1. W. Doster, S. Cusack, W. Petry, Nature 337 (1989) 754-756.

2. B. F. Rasmussen, A. M. Stock, D. Ringe, G. A. Petsko, Nature 357 (1992) 423-424.

3. A. L. Lee, A. J. Wand. Nature 411 (2001) 501-504.

4. J. H. Roh, V. N. Novikov, R. B. Gregory, J. E. Curtis, Z. Chowdhuri, A. P. Sokolov, Phys. Rev. Lett. 95 (2005) 038101-1-4.

5. K. Kawai, T. Suzuki, M. Oguni, Thermochim. Acta 431 (2005) 4-8.

6. K. Kawai, T. Suzuki, M. Oguni, Biophys. J. 90 (2006) 3732-3738.

7. Y. Miyazaki, T. Matsuo, H. Suga, J. Phys. Chem. B. 104 (2000) 8044-8052.

8. C. Bon, A. J. Dianoux, M. Ferrand, M. S. Lehmann, Biophys. J. 83 (2002) 1578-1588.

9. J. H. Roh, J. E. Curtis, S. Azzam, V. N. Novikov, I. Peral, Z. Chowdhuri, R. B. Gregory, A. P. Sokolov, Biophys. J. 91 (2006) 2573-2588.

10. S.-H. Chen, L. Liu, E. Fratini, P. Baglioni, A. Faraone, E. Mamontov, Proc. Natl. Acad. Sci. USA 103 (2006) 9012-9016.

11. P. Kumar, Z. Yan, L. Xu, M. G. Mazza, S. V. Buldyrev, S.-H. Chen, S. Sastry, H. E. Stanley, Phys. Rev. Lett. 97 (2006) 177802-1-4.

12. G. Sartor, A. Hallbrucker, E. Mayer, Biophys. J. 69 (1995) 2679-2694.

13. N. Miura, Y. Hayashi, N. Shinyashiki, S. Mashimo, Biopolymers 36 (1995) 9-16. 
14. J. Eden, P. R. C. Gascoyne, R. Pethig, J. Chem. Soc., Faraday Trans. 176 (1980) 426-434.

15. A. Yokoyama, H. Ishikawa, M. Shinohara, N. Shinyashiki, S. Yagihara, Y. Hayashi, Proceedings of Flow Dynamics: The Second International Conference on Flow Dynamics, American Institute of Physics CP832 (2006) 163-166.

16. N. Shinyashiki, W. Yamamoto, A. Yokoyama, T. Yoshinari, S. Yagihara, R. Kita, K. L. Ngai, S. Capaccioli, J. Phys. Chem. B. 113 (2009) 14448-14456.

17. J. E. Jentoft, R. G. Bryant, J. Am. Chem. Soc. 96 (1974) 297-299.

18. B. D. Hilton, E. Hsi, R. G. Bryant, J. Am. Chem. Soc. 99 (1977) 8483-8490.

19. E. R. Andrew, D. J. Bryant, E. M. Cashell, Chem. Phys. Lett. 69 (1980) 551-554.

20. T. G. Pedersen, B. W. Sigurskjold, K. V. Andersen, M. Kjær, F. M. Poulsen, C. M. Dobson, C. Redfield, J. Mol. Biol. 218 (1991) 413-426.

21. G.. Diakova, Y. A. Goddard, J. P. Korb, R. G. Bryant, J. Magn. Reson. 189 (2007) 166-172.

22. F. Mallamace, S. H. Chen, M. Broccio, C. Corsaro, V. Crupi, D. Majolino, V. Venuti, P. Baglioni, E. Fratini, C. Vannucci, H. E. Stanley, J. Chem. Phys. 127 (2007) 045104-1.

23. P. Neudecker, P. Lundström, L. E. Kay, Biophys. J. 96 (2009) 2045-2054.

24. B. Borah, R. G. Bryant, Biophys. J. 38 (1982) 47-52.

25. H. Peemoeller, F. G. Yeomans, D. W. Kydon, A. R. Sharp, Biophys. J. 49 (1986) 943-948.

26. L. T. Kakalis, T. F. Kumosinski, Biophys.Chem. 43 (1992) 39-49. 
27. M. G. Usha, J. Speyer, R. J. Wittebort, Chem. Phys. 158 (1991) 487-500.

28. J. W. Mack, M. G. Usha, J. Long, R. G. Griffin, R. J. Wittebort, Biopolymers 53 (2000) $9-18$.

29. Y. A. Goddard, J. -P. Korb, R. G. Bryant, Biophys. J. 91 (2006) 3841-3847.

30. K. Schmidt-Rohr, H. W. Spiess, Multidimensional Solid-State NMR and Polymers, Academic Press, London, 1994.

31. R.R. Vold, Deuterium NMR Studies of Dynamics in Solids and Liquid Crystals, in: R. Tycko (Ed.), Nuclear Magnetic Resonance Probes of Molecular Dynamics, Kluwer Academic Publishers, Norwell, MA, 1994, pp 27-112.

32. R. R. Vold, R. L. Vold, Deuterium Relaxation in Molecular Solids in: W. S. Warren (Ed.), Advances in Magnetic and Optical Resonance, Vol. 16, Academic Press Inc, San Diego, 1991, pp 85-171.

33. J.R. Long, R. Ebelhauser, R.G. Griffin, J. Phys. Chem. A. 101 (1997) 988-994.

34. J. Kimura, T. Fukase, M. Mizuno, M. Suhara, Z. Naturforsch. 53a (1998) 453-458.

35. M. Mizuno, Y. Hamada, T. Kitahara, M. Suhara, J. Phys. Chem. A 103 (1999) 4981-4985.

36. M. Mizuno, T. Iijima, M. Suhara, J. Phys. Condens. Matter. 12 (2000) 7261-7273.

37. T. Iijima, M. Mizuno, Chem. Phys. Lett. 380 (2003) 736-741.

38. M. Mizuno, Y. Suzuki, K. Endo, M. Murakami, M. Tansho, T. Shimizu, J. Phys. Chem. A. 
111 (2007) 12954-12960.

39. T. Araya, A. Niwa, M. Mizuno, K. Endo, Chem. Phys. 344 (2008) 291-298.

40. R. J. Wittebort, M. G. Usha, D. J. Ruben, D. E. Wemmer, A. Pines, J. Am. Chem. Soc. 110 (1988) 5668-5671.

41. M. Vogel, Phys. Rev. Lett. 101 (2008) 225701-1-4.

42. S. A. Lusceac, M. R. Vogel, C. R. Herbers, Biochim. Biophys. Acta. 1804 (2010) 41-48.

43. S. A. Lusceac, M. Vogel, J. Phys. Chem. B 114 (2010) 10209-10216.

44. N. Bloembergen, E. M. Purcell, R. Pound. Phys. Rev. 73 (1948) 679-712.

45. P. A. Beckmann, Phys. Rep. 171 (1988) 85-128. 


\section{FIGURE CAPTIONS}

Figure 1 Temperature dependence of ${ }^{2} \mathrm{H}$ NMR spectrum for BSA.

Figure 2 Partially relaxed ${ }^{2} \mathrm{H}$ NMR spectrum of BSA for inversion recovery method at $233 \mathrm{~K} . \tau_{r}$ shows the recovery time after a $180^{\circ}$ pulse.

Figure 3 Magnetization recovery for the inversion recovery method. (a) $253 \mathrm{~K}$, (b) $223 \mathrm{~K}$, (c) $183 \mathrm{~K}$. Solid lines show theoretical fitting of two stretched exponential functions of eq (1) in the text.

Figure 4 Temperature dependence of ${ }^{2} \mathrm{H}$ NMR mean spin-lattice relaxation time $\left\langle T_{1}\right\rangle$ for BSA. Solid and open circles show $\left\langle T_{1}\right\rangle$ for the fast component $\left(\left\langle T_{1 f}\right\rangle\right)$ and the slow component $\left(\left\langle T_{1 s}\right\rangle\right)$, respectively.

Figure 5 Temperature dependence of correlation time $\tau_{\mathrm{cc}}$ obtained from ${ }^{2} \mathrm{H}$ NMR $\left\langle T_{1}\right\rangle$ for the fast component.

Figure $6{ }^{2} \mathrm{H}$ NMR spectra of the fast component for BSA. $\tau_{r}$ shows the recovery time after saturation pulses. Solid lines are observed spectra. Broken lines are simulated spectra assuming motions of water molecules in Fig. 7.

Figure 7 Motion of water molecules used for simulation of the ${ }^{2} \mathrm{H}$ NMR spectrum. ${ }^{2} \mathrm{H}$ NMR spectrum around $200 \mathrm{~K}$ could be well reproduced by a combination of the $180^{\circ}$ flip and a slow jump to the other two sites in the tetrahedral sites (reorientation of water molecules).

Figure 8 Distribution of rates of the $180^{\circ}$ flip $k_{180^{\circ}}(\mathrm{a})$, rates of reorientation $k_{\mathrm{re}}(\mathrm{b})$ and angles $\theta$ 
(c) for water molecules. Red broken lines in (a) show distribution of the signal component affected by partial relaxation. 


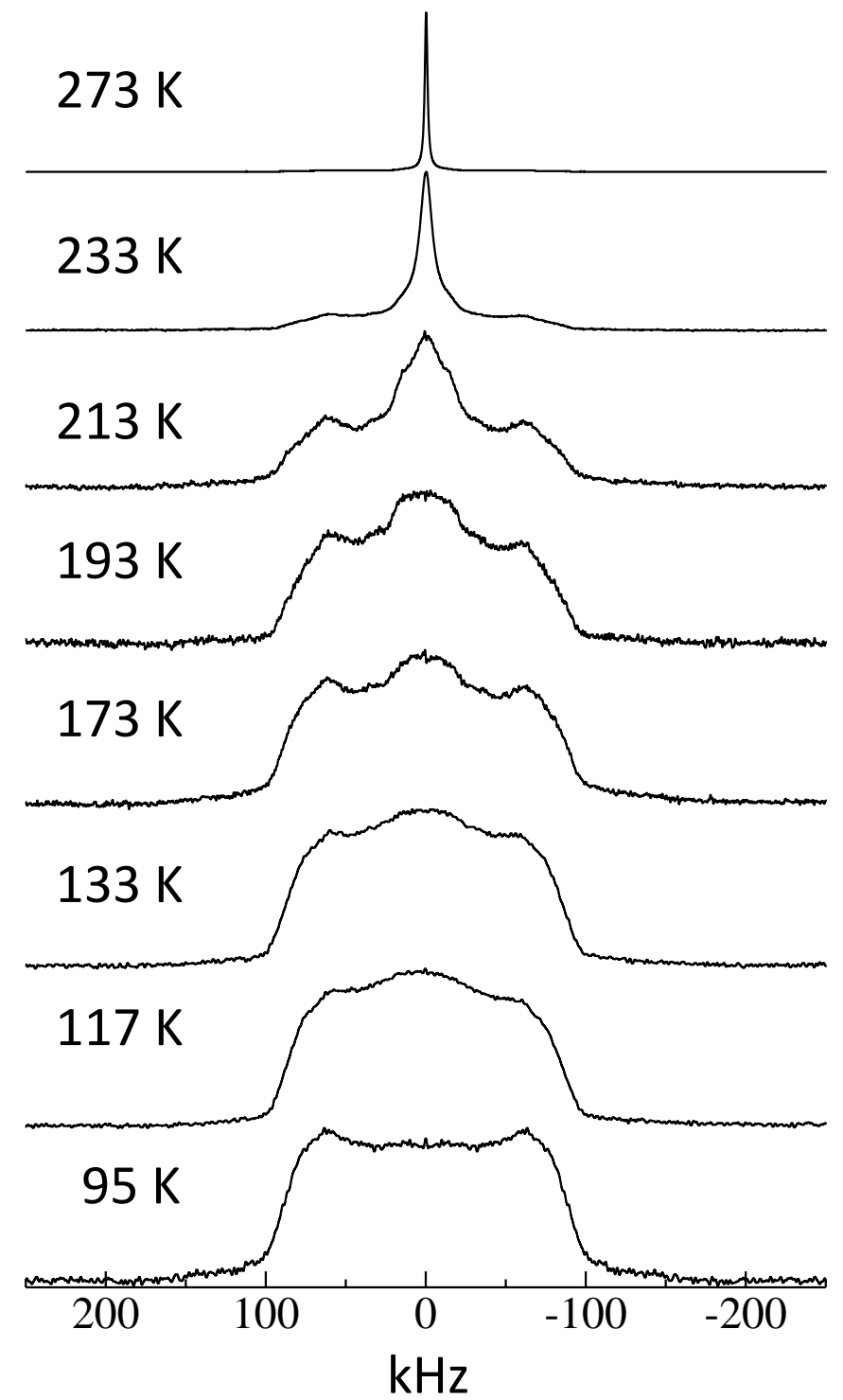

Fig.1 


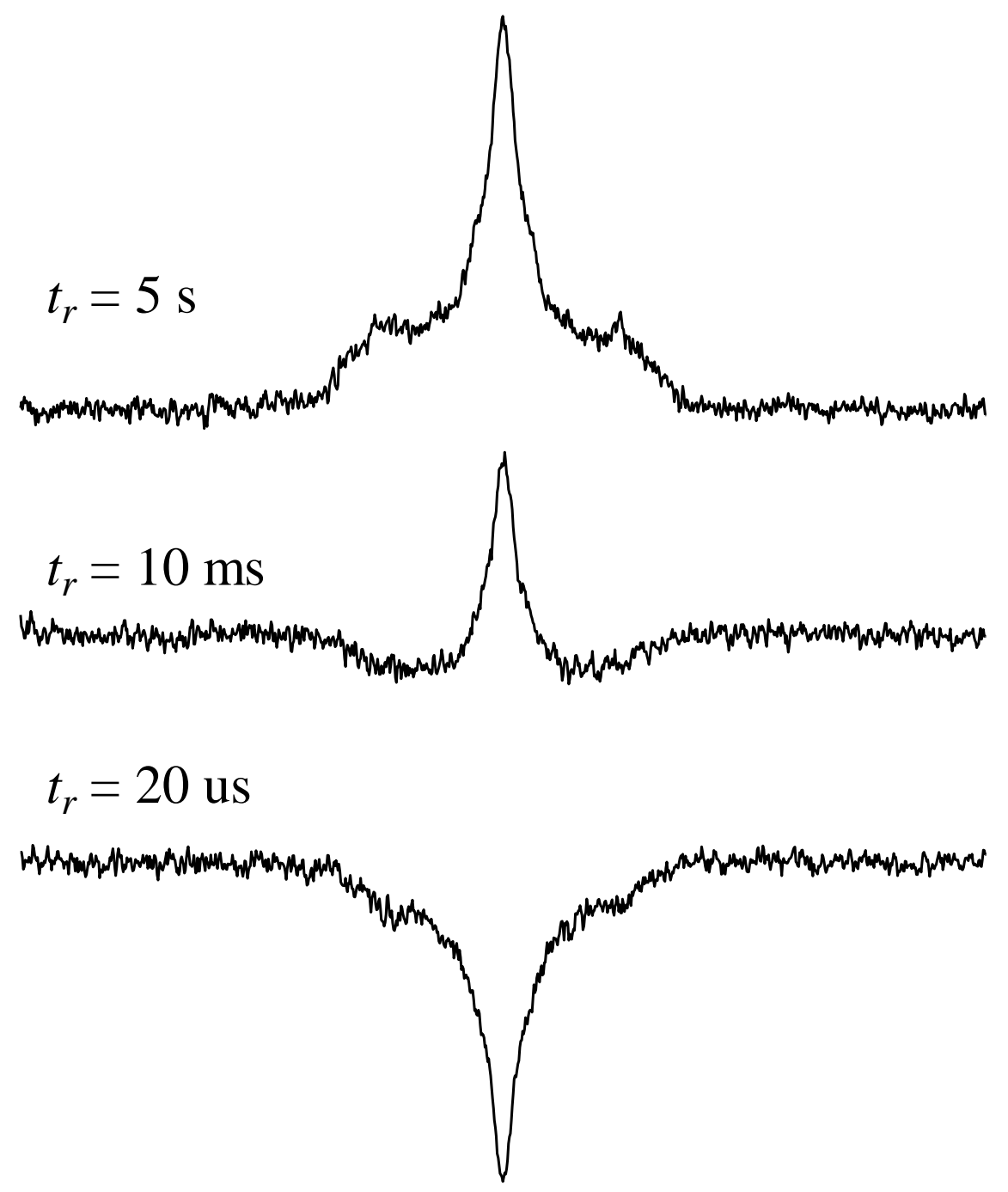

Fig. 2 


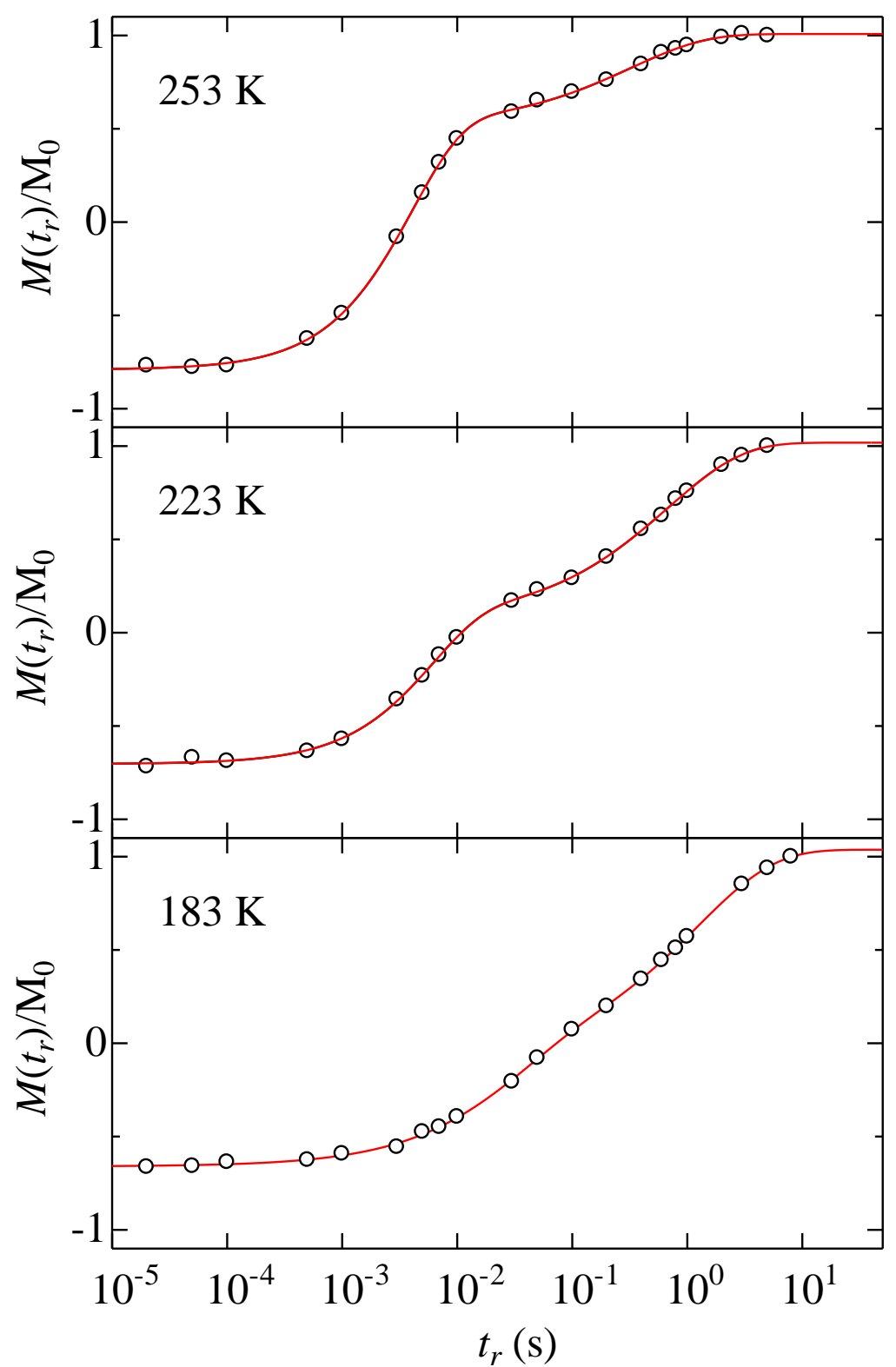

Fig. 3 


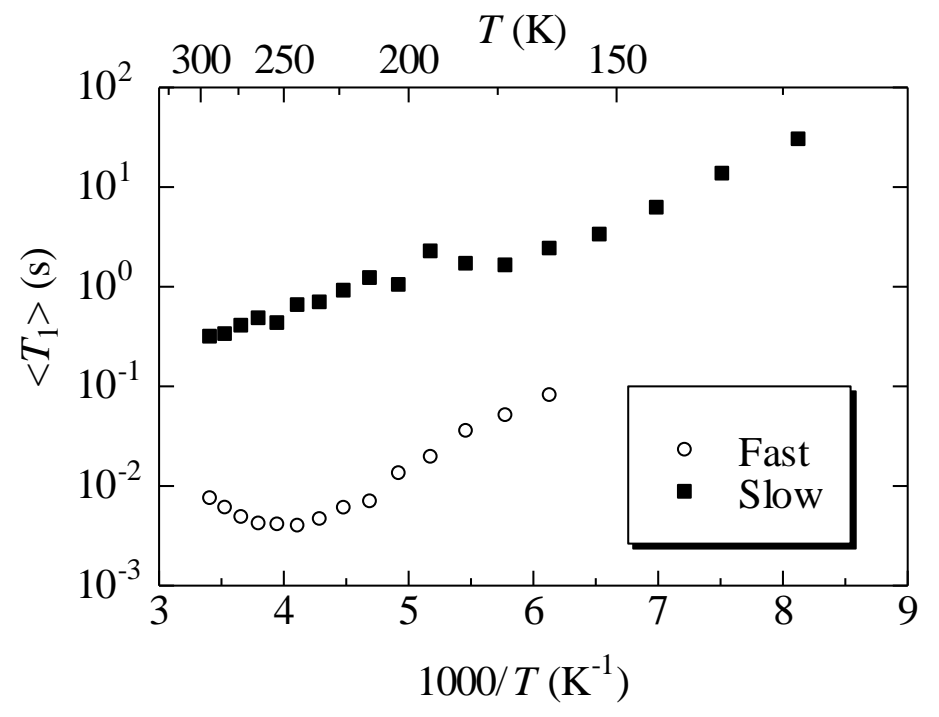

Fig. 4 


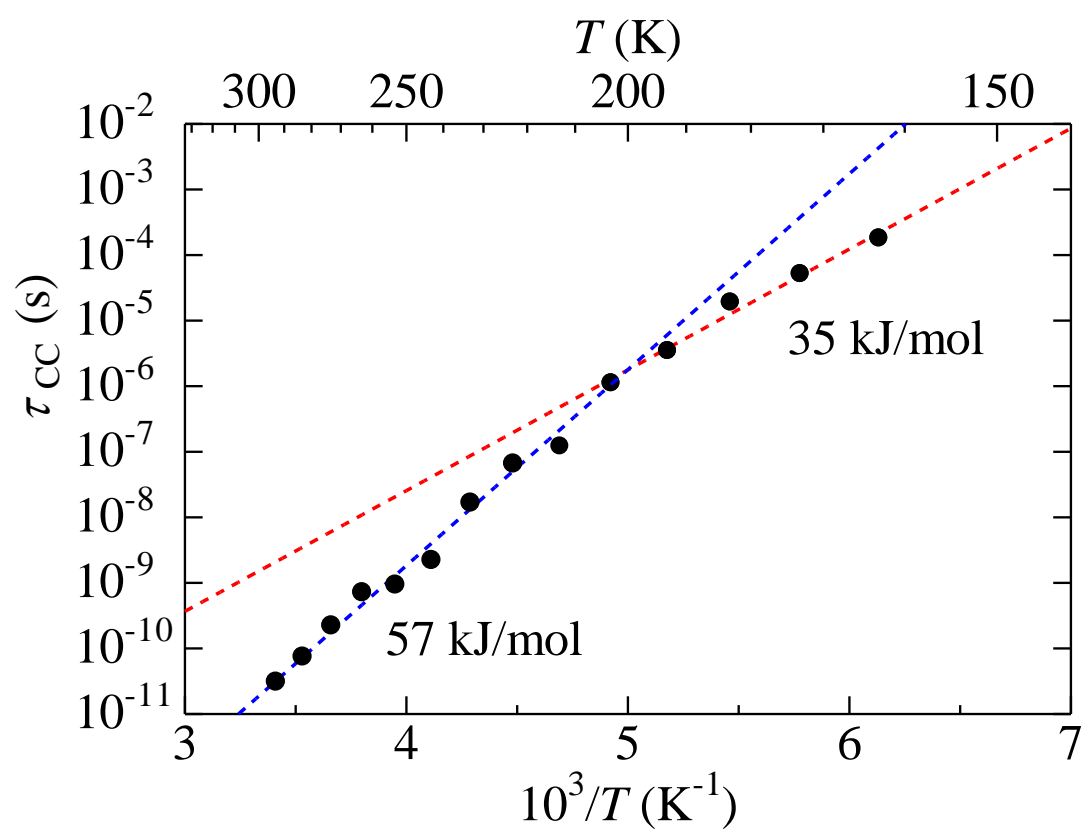

Fig.5 


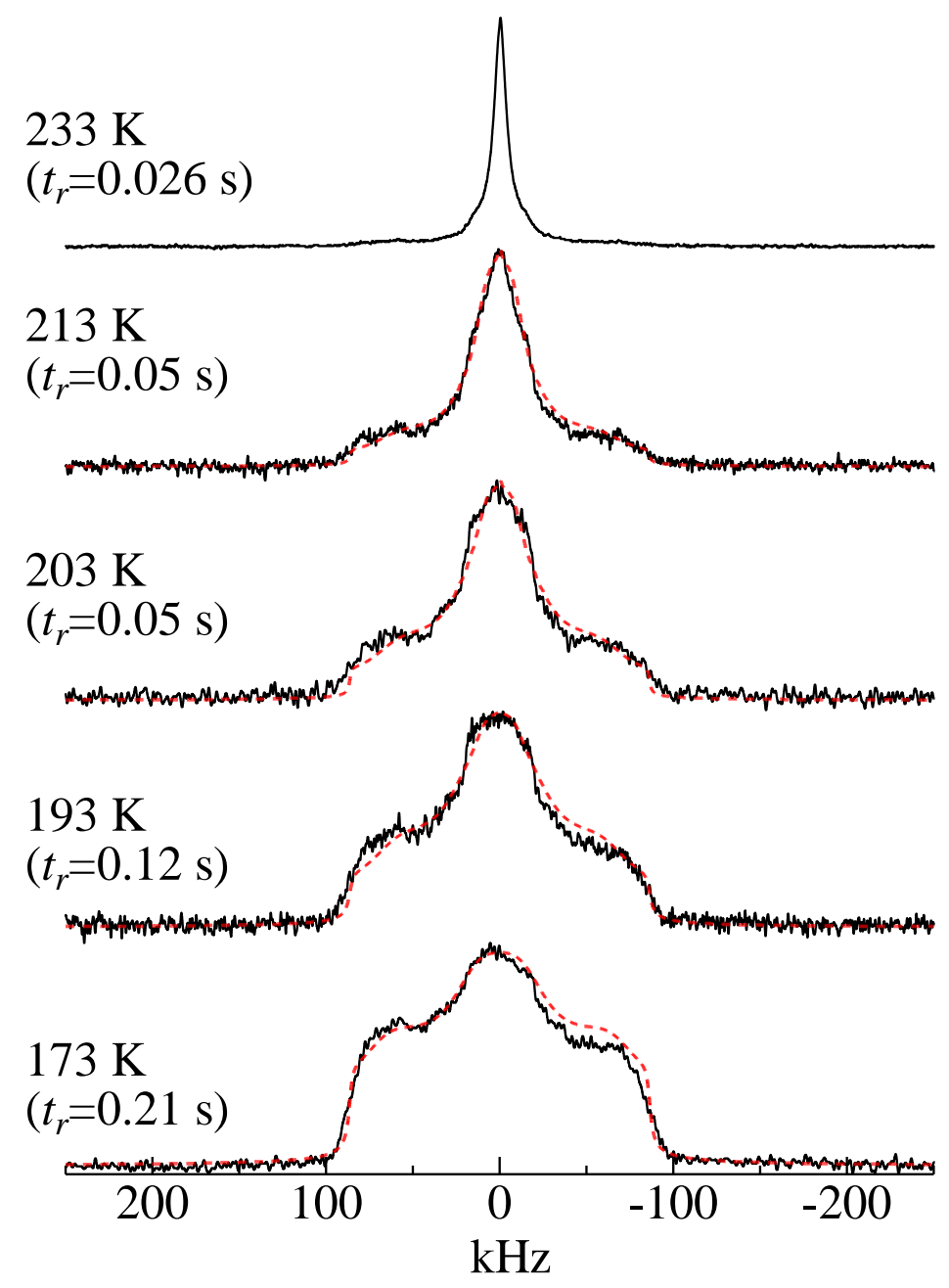

Fig. 6 


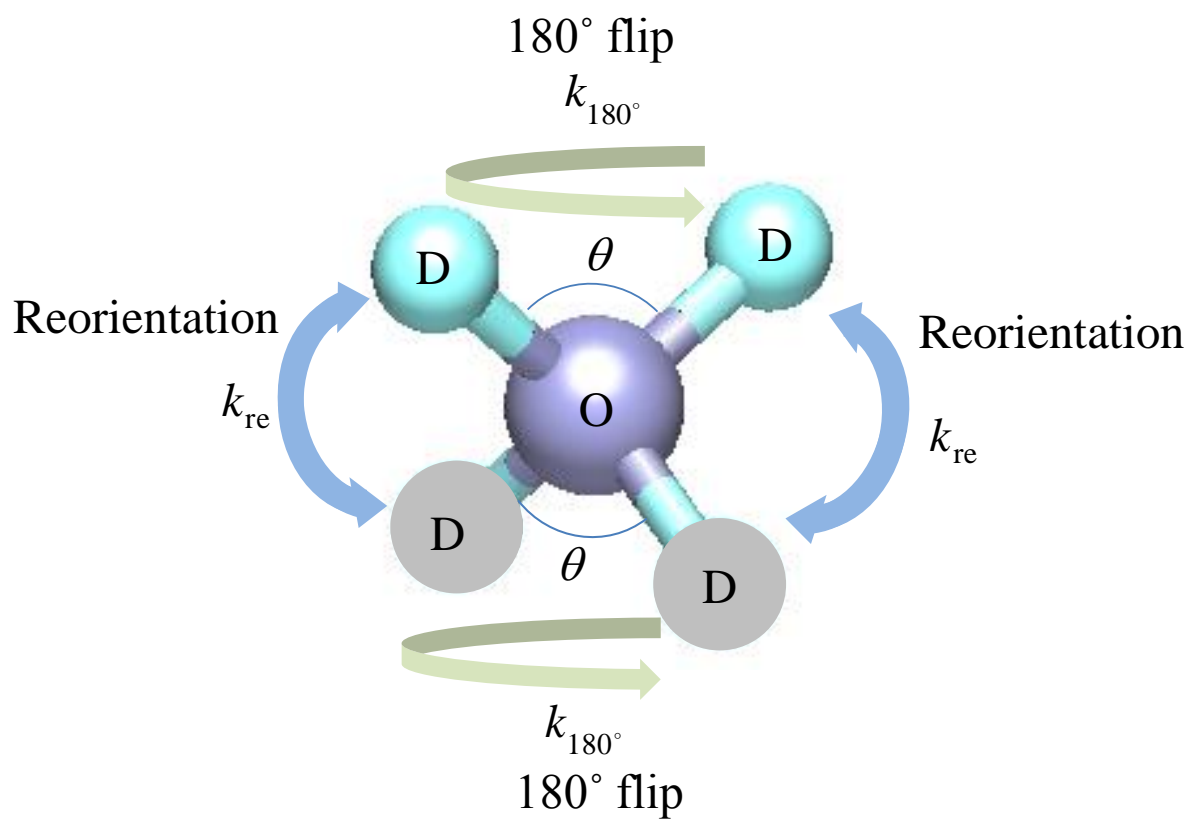

Fig. 7 
(a)

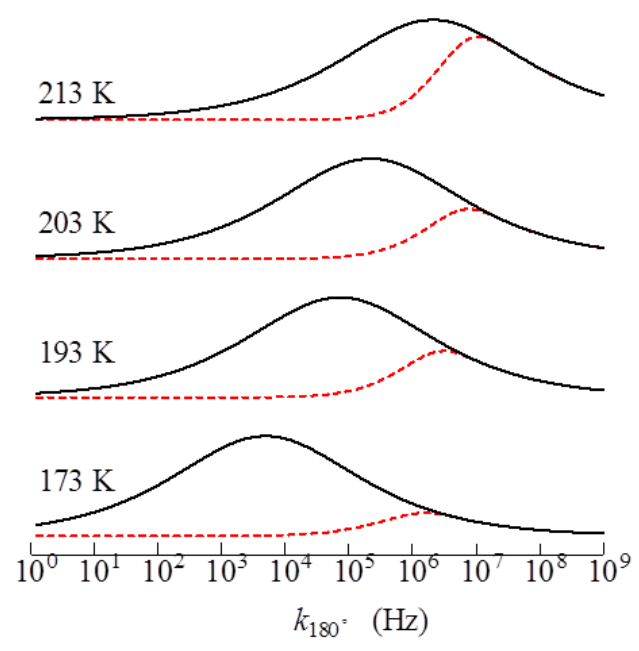

(b)
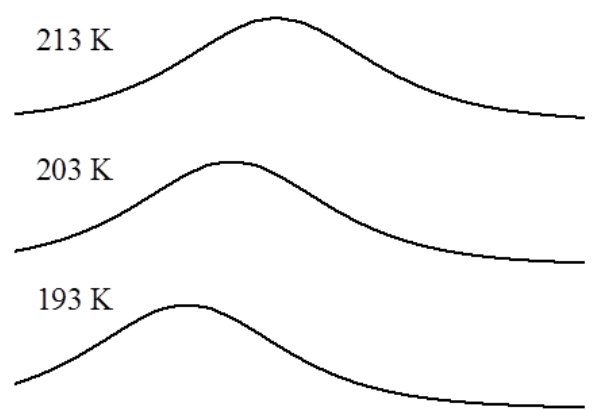

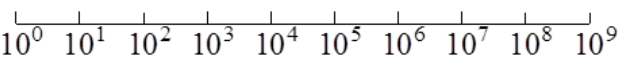
$k_{r e}(\mathrm{~Hz})$
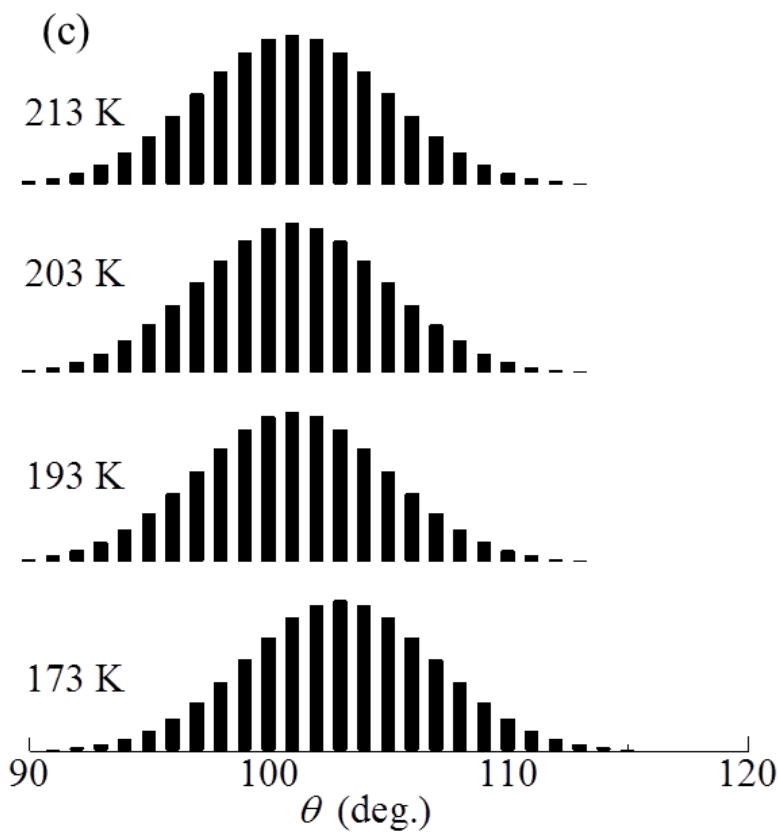

Fig. 8 